\title{
An Efficient Data Dissemination in Urban Vehicular Ad Hoc Networks
}

\author{
Shiow Fen Hwang ${ }^{1}$, Chia Hao $\mathrm{Chu}^{2}$, Chyi Ren Dow ${ }^{1}$ \\ ${ }^{1}$ Dept. of Information Engineering and Computer Science, Feng Chia University \\ No. 100,Wenhwa Rd., Seatwen, Taichung, Taiwan, R.O.C. \\ sfhwang@mail.fcu.edu.tw; crdow@fcu.edu.tw \\ ${ }^{2}$ Mars Semiconductor Corp \\ 3FC5, No. 1, Lihsin Rd., Science Park, Hsinchu, Taiwan, R.O.C. \\ pizzken@hotmail.com
}

\section{Extended Abstract}

With the rapid development of wireless technologies, vehicular ad hoc networks (VANETs) attract people's attention in recent years. VANETs consist of vehicles and some facilities which are deployed on the road sides. And each vehicle equips wireless communication devices to communicate with other vehicles and facilities. VANETs support a wide range of applications and have been extensively studied. Data dissemination is an important issue in VANETs. However, in order to avoid packet flooding, data usually be disseminated in some specific areas. Therefore, how to select an appropriate area to achieve effective data dissemination is more important. Although, there are many researches about the issue [1] [2] [3] and a lot of novel schemes are proposed [4] [5], they usually consider the location of event as the dissemination area. This leads to more control packets and delay time when vehicles do not know the dissemination area or need multiple event informations at the same time.

In this paper, we propose an efficient data dissemination in urban vehicular ad hoc networks which has better success ratio of data requests and less control packets. First, find an appropriate dissemination area and spread the information of events over the area. We select the intersection with the maximum traffic flow as the center of dissemination area. The size of the area is determined by the importance of events which are classified into three levels. The events with higher level are more important. The dissemination area of $i$-th level events is bounded by the intersections which are (i-1)-hop far from the center. Secondly, choose a path to query the event information and a path to return the data quickly. The request packet will be sent to the dissemination area by a shortest path. The first vehicle received the packet will reply the data according to the trajectory of vehicle that issued the request packet. In addition, due to the mobility of vehicles, we also present some mechanisms to ensure the delivery efficiently. Finally, we use Network Simulator 2.35 (NS2) to build simulation environments and compare the proposed method with CDN and CDNY [4]. The simulation results show that the proposed method outperforms CDN and CDNY in terms of the control packets, the delay and the packet delivery ratio.

\section{References}

[1] W. Chen, R. K. Guha, T. J. Kwon, J. Lee, and Y.Y. Hsu, "A survey and challenges in routing and data dissemination in vehicular ad hoc networks," in Proceedings of the IEEE International Conference on Vehicular Electronics and Safety, pp. 328-333, 2008.

[2] M. A Javed, J. Y. Khan, D. T. Ngo, "Multimedia Transmission for Emergency Services in VANETs," in Proceedings of the IEEE International Conference on Vehicular Technology, pp. 1-5, 2014.

[3] D. Jin, F. Shi, J. Song, "Cluster Based Emergency Message Dissemination Scheme for Vehicular Ad Hoc Networks," in Proceedings of the 9th International Conference on Ubiquitous Information Management and Communication, 2015, pp. 1-8.

[4] N. Nakamura, Y. Niimi and S. Ishihara, "Live VANET CDN: Adaptive data dissemination scheme for locationdependent data in VANETs," in Proceedings of the IEEE International Conference on Vehicular Networking (VNC), pp. 95-102, 2013. 
[5] Y. Niimi and S. Ishihara, "Demand map-based data dissemination scheme for location dependent data in VANETs," in Proceedings of the International Conference on Mobile Computing and Ubiquitous Networking (ICMU), pp. 9697, 2015. 